\title{
Pilot Scheduling to Mitigate Pilot Contamination using Optimization Algorithm in Massive MIMO Systems
}

\author{
Mukund B. Wagh \\ Department of Computer Engineering, \\ Sinhgad College of Engineering, Pune, Maharashtra, India \\ mukundbaburaowagh@gmail.com
}

\author{
Nilesh G. Bhojne \\ Assistant Professor, \\ Department of Computer Engineering, \\ Sinhgad College of Engineering, Pune. India
}

\begin{abstract}
In wireless communication systems, Massive-MIMO shows a potential method to provide enhanced spectral proficiency, link reliability and present numerous users. Here, the most important objective is to resolve the pilot contagion problem in massive-MIMO systems. Hence, this work uses two techniques to reduce contagion. The user grouping method is presented based on the sparse FCM is presented that groups user parameters namely SINR, large-scale fading factor, and user distance. Moreover, to the center users, a similar pilot series is allocated wherein the pilot contagion impact is restricted when the technique allocates the orthogonal pilot series to edge users that undergo relentlessly from pilot contagion. Hence, developed user grouping shuns from an unsuitable grouping of users, set up the effectual grouping still in most awful circumstances of the channel. Then, pilot scheduling is carried out on basis of the Binary Particle Swarm Optimization (BPSO). Finally, the pilot scheduling performance based on the grouping-based ESMO is calculated on the basis of the attainable rate and SINR. The developed technique attains the maximum achievable rate and the maximum SINR.
\end{abstract}

Keywords: MIMO; User Grouping, Channel; FCM, Pilot Sequences, Optimization Methods

\begin{tabular}{ll} 
Nomenclature & \\
\hline Abbreviation & \multicolumn{1}{c}{ Description } \\
\hline Massive-MIMO & Massive Multiple-input Multiple-output \\
MS & Mobile Station \\
BS & Base Station \\
UTs & User Terminals \\
MU & Multi-User \\
AWGN & Additive White Gaussian Noise \\
FPR & Fractional Pilot Reuse \\
LU & Legitimate User \\
SINRs & Signal-to-Interference-Plus-Noise Ratios \\
FCM & Fuzzy C-Means Clustering \\
KDP & Key Disagreement Probability \\
PCA & Pilot Contamination Attack \\
CSI & Channel State Information \\
Eve & eavesdropper \\
\hline
\end{tabular}

\section{Introduction}

In MS, the massive MIMO system is operational in a huge number of antennas at the Base Station and a single antenna. In a characteristic MIMO system, the massive MIMO system has a lot of benefits, such as enhanced link reliability, spectral efficiency, and minimized interference among users [1].

In recent times, the center of attention has shifted to MU massive MIMO systems, wherein a Base Station concurrently serves multiple mobile stations [2]. Subordinate equipment costs, and superior multi-user diversity, are a number of benefits of MU massive MIMO systems. At the transmitter, CSI is necessary to attain the benefits of massive MIMO. In general, CSI can be calculated by means of the aid of pilot series in wireless networks. The channel estimation precise directly affects massive MIMO performance [4]. 
In a multi-cell network, while users exploit similar or nonorthogonal pilots in diverse cells, the Base Station will calculate an overlapped CSI from these users. Hence, the pilot contamination is referred to as the precoder based on the calculated CSI will produce interference to these users [1]. To enhance the massive MIMO system performance the pilot contamination has to turn out to be a bottleneck.

For the massive MIMO, the conventional techniques for minimization of pilot contamination are designed wherein the pilots acquire time-multiplexed with the data, henceforth it is called as timemultiplexed pilots. Moreover, the data to channel vectors, the autocorrelation matrix has been seen in eigenvectors are attained. For the channel estimation technique, the preferred users are proposed by exploiting the observation. By means of channel vectors, the projecting contaminated channel estimate is done by the pilot decontamination in an interference-free subspace spanned of preferred users, while the asymptotic circumstances for the separability among subspaces of preferred and the interfering users. For pilot allocation, the coordinated technique is proposed to separate interfering and preferred users in correlated channels [11].

The most important objective of this work is to develop a user grouping method by exploiting the developed sparse FCM. This technique groups the users based on the parameters namely SINR, largescale fading factor, and user distance to allocate the similar pilot series, in-center users. Hence, the developed user grouping shuns from the unsuitable users grouping, set up the effectual grouping even in the most awful circumstances of the channel.

\section{Literature Survey}

In 2020, Parisa Pasangi et al [1], developed a blind technique to estimate the downlink channel increase in TDD-based multi-cell MU massive MIMO system in attendance of pilot contagion. For downlink received signal the average energy was exhibited in the developed technique for every user which was a function of the channel increase. Hence, this average energy was computed by exploiting the received signal samples, and the gain of the channel was calculated.

In 2019, Awais Ahmed et al [2], presented a new secret key generation technique in attendance of an active Eve from general arbitrariness of frequency selective channel among legal nodes. From Eve, the PCA relentlessly impedes secret key generation from the estimation of the reciprocal channel among lawful nodes. The two-step channel estimation model was presented to alleviate the PCA impact in the pilot stage whereas decoded data was exploited as a reference to estimation of the channel. In the attendance of signal contagion, the developed technique attains minimum KDP.

In 2020, Muhammad Hassan et al [3], proposed a minimum-complexity PCA detector to a multi-cell MU-MIMO system. In the reference cell, an active Eve transfers synchronized training series of the LU in attack to change the precoder to steal information in the downlink data stage. The binary hypotheses were formulated to detect the active Eve from the contagion estimation of the channel.

In 2018, Jiancun Fan et al [4], developed an FPR model to alleviate pilot contagion in a MIMO system. Users were classified in FPR into cell-center and cell-edge ones based on their SINRs during the receiver side. Subsequently, to evade strong interference from neighboring cells cell-edge users by means of minimum SINRs in diverse cells exploit orthogonal pilots or diverse pilots.

In 2020, Satyasen Panda [5], investigated the downlink and uplink single-cell Massive MIMO system performance in channel deviating circumstances because of speedy user mobility. For CSI estimation the mobile users transfer orthogonal pilot series to the BS prior to transmission of data during uplink. By exploiting appropriate numerical analysis the SINR and mean spectral efficiency were estimated in both scenarios.

\section{System Model}

The system model of massive MIMO is explained in this section. The massive MIMO model is described in Fig 1 that consists of antenna arrays, BS and the number of user terminals. The data streams are transmitted by BS from the diverse antenna into several terminals at a similar frequency. 


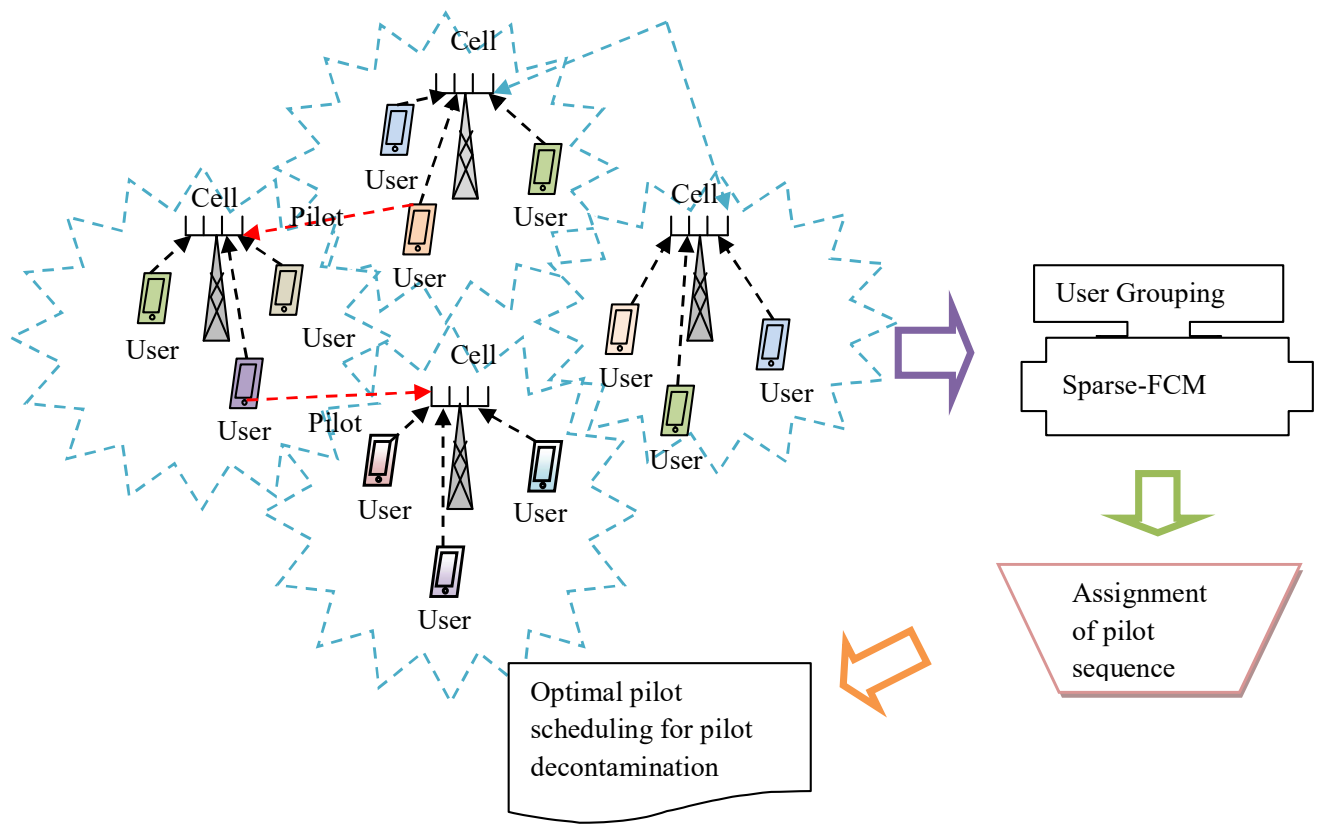

Fig. 1. System model of MIMO

In order to deliver the enhanced coverage, the massive MIMO is used and maximum rates, energy effectual and the link reliability however the massive MIMO occurs numerous confronts in terms of the pilot design and channel estimation that minimizes massive MIMO system performances.

Therefore, an effective method is developed in order to alleviate the pilot contagion to resolve the pilots' problem. The central BS is exploited by each hexagonal cell and it is linked with B antennas for $\mathrm{V}$ user's (V >> B) single-antenna users. Moreover, channel vector $\mathrm{a}_{\mathrm{bcv}} \in \mathrm{G}^{\mathrm{B} \times 1}$ indicates link straddling from $v^{\text {th }}$ user of $c^{\text {th }}$ cell to BS of $b^{\text {th }}$ cell that is indicated by,

$$
\mathrm{a}_{\mathrm{bcv}}=\mathrm{g}_{\mathrm{bcv}} \sqrt{\beta_{\mathrm{bcv}}}
$$

whereas, $\mathrm{g}_{\mathrm{bcv}}$ indicates the small fading vector whereas $\mathrm{g}_{\mathrm{bcv}} \in \mathrm{G}^{\mathrm{B} \times 1}$, the shadow fading, and path loss, is indicated by $\alpha_{a b u}$ and it is stated below,

$$
\beta_{\mathrm{bcv}}=\left(\frac{\mathrm{U}_{\mathrm{bcv}}}{\mathrm{h}_{\mathrm{bcv}} / \mathrm{R}}\right)^{\alpha}
$$

whereas, shadow fading is indicated by $\mathrm{U}_{\mathrm{bcv}}$, and $\mathrm{h}_{\mathrm{bcv}}$ denotes distance calculated among $\mathrm{v}^{\text {th }}$ user in $c^{\text {th }}$ cell and BS in $b^{\text {th }}$ cell. The cell radius is denoted as $\mathrm{R}$. Hence, matrix id produced to represent channel among all V users in $\mathrm{c}^{\text {th }}$ cell, and Base Station in $\mathrm{c}^{\text {th }}$ cell is defined as,

$$
\mathrm{N}_{\mathrm{cd}}=\mathrm{J}_{\mathrm{cd}} \mathrm{K}_{\mathrm{cd}}^{\frac{1}{2}}
$$

whereas, the coefficient matrix of fast fading is denoted as $J_{c d} \in G^{B \times V}$ that is situated among $B$ antennas of Base Station present in the $d^{\text {th }}$ cell, and V users in $c^{\text {th }}$ cell, and $D_{\text {cd }} \in G^{\mathrm{V} \times \mathrm{V}}$ denotes the diagonal matrix by means of diagonal elements $\left[\mathrm{D}_{\mathrm{cd}}\right]_{\mathrm{vv}}=\beta_{\mathrm{cdv}}$. At coherence time, the channel vector is constant and changes autonomously in several coherence intervals. The downlink channel is generated by BS based on the uplink pilot series in coherence time in massive MIMO systems. During the data transmission, each and every user transfers uplink data to Base Stations and received in BS at $b^{\text {th }}$ cell is stated as,

$$
\mathrm{k}_{\mathrm{b}}^{1}=\sqrt{\mathrm{A}_{1}} \sum_{\mathrm{c}=1 \mathrm{v}=1}^{\mathrm{C}} \sum_{\mathrm{bsv}}^{\mathrm{v}} \mathrm{p}_{\mathrm{cv}}^{1}+\mathrm{n}_{\mathrm{b}}^{1}
$$

whereas, the variable broadcast from $v^{\text {th }}$ user in $c^{\text {th }}$ cell is stated as $p_{\mathrm{cv}}^{1}$ with $\in\left\{\left|p_{\mathrm{cv}}^{1}\right|^{2}\right\}=1$, and the uplink transmitter is stated as $\mathrm{A}_{1}$, and the expectation operator is stated as $\in(\cdot), \mathrm{n}_{\mathrm{b}}^{1} \in \mathrm{G}^{\mathrm{B} \times 1}$ states the 
AWGN vector with $\in\left\{n_{b}^{1}\left(n_{b}^{1}\right)^{H}\right\}=\eta_{n}^{2} H_{B}$, $\eta$ denotes the covariance, and $H_{B}$ denotes identity matrix of size $\mathrm{B} \times \mathrm{B}$. In BS, the pilot series matrix received at $\mathrm{b}^{\text {th }}$ cell is stated as,

$$
\mathrm{k}_{\mathrm{b}}^{\mathrm{r}}=\sqrt{\mathrm{A}_{\mathrm{r}}}\left(\sum_{\mathrm{c}=1}^{\mathrm{C}} \mathrm{N}_{\mathrm{bc}} \theta_{\mathrm{b}}\right)+\mathrm{n}_{\mathrm{b}}^{\mathrm{r}}
$$

whereas, $k_{b}^{r} \in G^{B \times \delta}$ when the time period of pilot series is stated as $\delta$, and $\theta_{b}=\left(\theta_{1}, \theta_{2}, \ldots, \theta_{V}\right)^{\mathrm{Y}}$ signifies transmitted pilot series at power constraint $\theta_{b} \theta_{b}^{N}=H_{v}$, and pilot transmit power is stated as $A_{r}$, and uplink channel noise vector which fits in to $G^{B \times \delta}$ is denoted as $n_{b}^{r}$. In this scenario, the Base Station of $\mathrm{b}^{\text {th }}$ cell calculates the channel matrix $\mathrm{N}_{\mathrm{bb}}$ by exploiting convolutional channel,

$$
\hat{\mathrm{N}}_{\mathrm{bb}}=\frac{1}{\sqrt{\mathrm{A}_{\mathrm{r}}}} \mathrm{k}_{\mathrm{b}}^{\mathrm{r}} \theta_{\mathrm{b}}^{\mathrm{N}}=\mathrm{M}_{\mathrm{bb}}+\sum_{\mathrm{c} \neq \mathrm{b}} \mathrm{N}_{\mathrm{bc}}+\frac{1}{\sqrt{\mathrm{A}_{\mathrm{r}}}} \mathrm{n}_{\mathrm{b}}^{\mathrm{r}} \theta_{\mathrm{b}}^{\mathrm{M}}
$$

whereas, the linear integration of the channel $\mathrm{a}_{\mathrm{bcv}}$ for $1 \leq \mathrm{c} \leq \mathrm{C}$ is stated as $\mathrm{a}_{\mathrm{bbv}}$, that facades the channel users in the another cell linked by means of pilot series. This event is referred to as pilot contagion. Subsequently, from the $\mathrm{v}^{\text {th }}$ user the identified variable attained in $b^{\text {th }}$ which is devised as,

$$
\begin{aligned}
& \hat{\mathrm{p}}_{\mathrm{bv}}^{1}=\mathrm{a}_{\mathrm{bbv}}^{\mathrm{N}} \mathrm{K}_{\mathrm{b}}^{1} \\
& \hat{\mathrm{p}}_{\mathrm{bv}}^{1}=\left(\sum_{\mathrm{c}=1}^{\mathrm{C}} \mathrm{a}_{\mathrm{bcv}}^{\mathrm{M}}+\mathrm{m}_{\mathrm{bv}}^{\mathrm{N}}\right)\left(\sqrt{\mathrm{A}_{1}} \sum_{\mathrm{c}=1 \mathrm{v}=1}^{\mathrm{C}} \sum_{\mathrm{bcv}}^{\mathrm{v}} \mathrm{b}_{\mathrm{cv}}+\mathrm{n}_{\mathrm{b}}^{1}\right) \\
& \hat{\mathrm{p}}_{\mathrm{bv}}^{1}=\sum_{\mathrm{c}=1}^{\mathrm{C}} \mathrm{a}_{\mathrm{bcv}}^{\mathrm{N}} \cdot \sqrt{\mathrm{A}_{1}} \sum_{\mathrm{a}=1 \mathrm{v}=1}^{\mathrm{C}} \sum_{\mathrm{b}}^{\mathrm{V}} \mathrm{a}_{\mathrm{bcv}} \mathrm{p}_{\mathrm{cv}}^{1}+\gamma_{\mathrm{b}, \mathrm{v}}^{1} \\
& \hat{\mathrm{p}}_{\mathrm{bv}}^{1}=\sqrt{\mathrm{A}_{1}}\left(\mathrm{a}_{\mathrm{bbv}}^{\mathrm{N}} \mathrm{a}_{\mathrm{bbv}} \mathrm{p}_{\mathrm{bv}}^{1}+\sum_{\mathrm{c} \neq \mathrm{b}} \mathrm{a}_{\mathrm{bcv}}^{\mathrm{N}} \mathrm{a}_{\mathrm{bcv}} \mathrm{p}_{\mathrm{cv}}^{1} \cdot\right)+\gamma_{\mathrm{b}, \mathrm{v}}^{1} \\
& \hat{\mathrm{p}}_{\mathrm{bv}}^{1}=\mathrm{B} \sqrt{\mathrm{A}_{1}}\left(\beta_{\mathrm{bbv}} \mathrm{p}_{\mathrm{bv}}^{1}+\sum_{\mathrm{c} \neq \mathrm{b}} \beta_{\mathrm{bcv}} \mathrm{p}_{\mathrm{cv}}^{1} \cdot\right)+\gamma_{\mathrm{b}, \mathrm{v}}^{1}
\end{aligned}
$$

whereas, the variable $\mathrm{m}_{\mathrm{bv}}$ indicates $\mathrm{v}^{\text {th }}$ column of $\frac{\mathrm{n}_{\mathrm{b}}^{\mathrm{r}} \theta_{\mathrm{b}}^{\mathrm{N}}}{\sqrt{\mathrm{K}_{\mathrm{q}}}}$, and the variable $\gamma_{\mathrm{b}, \mathrm{v}}^{1}$ indicates orthogonality among the channels of numerous users. While the BS antennas number attains the infinity subsequently, the recognized variable is stated as,

$$
\mathrm{p}_{\mathrm{bv}}^{1} \approx \mathrm{B} \sqrt{\mathrm{A}_{\mathrm{l}}}\left(\beta_{\mathrm{bbv}} \mathrm{p}_{\mathrm{bv}}^{1}+\sum_{\mathrm{c} \neq \mathrm{j}} \beta_{\mathrm{bcv}} \mathrm{p}_{\mathrm{cv}}^{1}\right)
$$

\subsection{Parameters of User Computing}

In a massive MIMO system, the calculating parameters utilized are SINR, large-scale fading factor, and the distance of user [6]. To model user grouping technique, these parameters are crucial to enhance the system capacity, and the explanations of the user calculating parameters, are stated as follows,

User SINR: in the cell, the uplink SINR of the user is stated as,

$$
\operatorname{SINR}_{b v}^{1}=\frac{\left|a_{b b v}^{N} a_{b b v}\right|^{2}}{\sum_{c \neq b}\left|a_{b c v}^{N} a_{b c v}\right|^{2}+\frac{\left|\gamma_{b, b}^{1}\right|^{2}}{A_{1}}}
$$

Suppose the BS number tends to $\infty$, subsequently uplink SINR is stated as

Hence, user SINR is stated as $\mathrm{f}_{1}$.

$$
\operatorname{SINR}_{\mathrm{bv}}^{1}=\frac{\beta_{\mathrm{bbv}}^{2}}{\sum_{\mathrm{c} \neq \mathrm{b}} \beta_{\mathrm{bcv}}^{2}}
$$

\section{User large scale fading factor:}

In eq. (2), a large scaling factor of $v^{\text {th }}$ user of $c^{\text {th }}$ cell to Base Station of $b^{\text {th }}$ cell is derived. The large scale fading factor is stated as $\mathrm{f}_{2}$.

User distance: Among the position of $\mathrm{v}^{\text {th }}$ user and the center of the cell the distance of user is calculated. Therefore, the distance of user is calculated as,

$$
\mathrm{B}=\sqrt{\sum_{\mathrm{v}=1}^{\mathrm{U}}\left(\mathrm{w}_{\mathrm{v}}^{\text {loc }}, \mathrm{z}^{\text {cell }}\right)}
$$


Where as, the position of the $\mathrm{v}^{\text {th }}$ user is stated as $\mathrm{w}_{\mathrm{v}}^{\text {loc }}$, and the cell center is stated as $\mathrm{z}^{\text {cell }}$. The distance of the user is stated as $\mathrm{f}_{3}$. Therefore, the parameters of user grouping are stated as,

$$
\mathrm{T}=\mathrm{f}_{1}, \mathrm{f}_{2}, \mathrm{f}_{3}
$$

\section{Developed User Grouping to Reduce Pilot Contamination}

The developed user grouping approach on the basis of the sparse FCM [7] is stated in this section. Moreover, the grouping of the user is on the basis of 3 parameters and it is most important to comprehend that the grouping of the user contains impacts on pilot scheduling. Hence, the adopted grouping of users shuns from the unsuitable grouping of users, set up the effectual grouping in most awful circumstances of the channel.

\subsection{Massive MIMO System}

It represents a communication system in that Base Station with a small amount of antenna array serves numerous 10's of UTs, every antenna comprising of the single antenna in similar time-frequency resource [8]. The time-frequency resources need downlink pilots scale by means of antennas, hence a massive-MIMO system comprises numerous resources than the existing system [9].

\subsection{Sparse FCM for User Grouping}

The user grouping is performed exploiting sparse FCM that group users on basis of the parameters, namely SINR, large-scale fading factor, and the distance of the user. The sparse FCM steps are stated as follows,

i) Initialization: The initialization is the first step of the feature weights which is stated as,

$$
\varpi=\varpi_{1}^{\circ}=\varpi_{2}^{0}=\ldots=\varpi_{\mathrm{q}}^{\mathrm{o}}=\frac{1}{\sqrt{\mathrm{q}}}
$$

whereas, $q$ indicates the features, and the $\square$ attribute weights, signifies $q^{\text {th }}$ feature is stated as $\varpi_{q}^{e}$.

ii) Partition matrix updation: Let the cluster centers are fixed and attribute weights as, c and $\varpi$, correspondingly. The constraint is indicated as, $\sigma(z)$ that must be reduced if and only if,

$$
\mathrm{W}_{\mathrm{il}}=\left\{\begin{array}{lll}
\frac{1}{\mathrm{U}_{1}} & ; & \text { if } \mathrm{s}_{\mathrm{il}}=0 \text { and } \mathrm{U}_{1}=\text { cardinality }\left\{\mathrm{e}: \mathrm{s}_{\mathrm{il}}=0\right\} \\
0 & ; \quad \text { if } \mathrm{s}_{\mathrm{il}} \neq 0 \text { but } \mathrm{s}_{\mathrm{iy}}=0 \text { for some } \mathrm{y}, \mathrm{y} \neq \mathrm{l} \\
\sum_{\mathrm{y}=1}^{\mathrm{m}}\left(\frac{\mathrm{s}_{\mathrm{il}}}{\mathrm{s}_{\mathrm{yl}}}\right)^{\left(\frac{1}{\beta-1}\right)} & ; & \text { Otherwise }
\end{array}\right.
$$

whereas, $\mathrm{s}_{\mathrm{il}}=\sum_{\mathrm{l}=1}^{\mathrm{m}} \varpi_{\mathrm{e}}\left(\mathrm{v}_{\mathrm{ei}}-\mathrm{c}_{\mathrm{li}}\right)^{2}$ and Cardinality $(\mathrm{A})$ indicates the cardinality of the set $\mathrm{A}$. The distance measure is indicated as, $\mathrm{s}_{\mathrm{il}}$ which denotes the distance among the $\mathrm{i}^{\text {th }}$ node regarding the $\mathrm{l}^{\text {th }}$ cluster center.

iii) Cluster centers formulation: In order to compute the cluster centers, it is very important to fix attribute weight $\varpi$ and a data matrix $Z$, and calculation of cluster center is stated as,

$$
\mathrm{y}_{\text {le }}=\left\{\begin{array}{lll}
0 & ; & \text { if } \varpi_{\mathrm{e}}=0 \\
\sum_{\mathrm{i}=1}^{\mathrm{n}} \mathrm{W}_{\mathrm{il}}^{\beta} \cdot \mathrm{v}_{\mathrm{ei}} & ; & \text { if } \varpi_{\mathrm{e}} \neq 0 \\
\sum_{\mathrm{i}=1}^{\mathrm{n}} \mathrm{W}_{\mathrm{il}}^{\beta} &
\end{array}\right.
$$


whereas $\mathrm{l}=1, \ldots, \mathrm{v}$ and $\mathrm{e}=1, \ldots, \mathrm{d}$. The index lindicates cluster center and e indicates data attribute. The $\mathrm{e}^{\text {th }}$ feature response to the objective model is indicated as, $\varpi_{\mathrm{e}}$ and constant Z indicates dissimilarity measure.

iv)Class derivation: On the basis of fixed clusters $\mathrm{y}=\left\{\mathrm{y}_{1}, \mathrm{y}_{2}, \ldots, \mathrm{y}_{1}, \ldots, \mathrm{y}_{\mathrm{m}}\right\}$ the class is identifies with membership $\mathrm{W}$. The class $\mathrm{Y}_{\mathrm{e}}$ is decided by exploiting the objective model which is stated as,

$\max _{\varpi} \sum_{\mathrm{e}=1}^{\mathrm{d}} \varpi_{\mathrm{e}} . \mathrm{Y}_{\mathrm{e}}$, so that $\|\varpi\|_{2}^{2} \leq 1,\|\varpi\|_{\kappa}^{\kappa} \leq \lambda$ produces $\varpi^{*}$.

whereas, $\lambda$ indicates the tuning parameter and $(0 \leq \kappa \leq 1) ;\|\omega\|_{\kappa}^{\kappa}=\sum_{e=1}^{d}\left|\varpi_{e}\right|^{\kappa}$.

v) Terminate: The iteration is sustained till iteration attains utmost number. The halting criteria is decided as,

$$
\frac{\sum_{\mathrm{e}=1}^{\mathrm{q}}\left|\varpi_{\mathrm{e}}^{*}-\varpi_{\mathrm{e}}^{\mathrm{o}}\right|}{\sum_{\mathrm{e}=1}^{\mathrm{q}}\left|\varpi_{\mathrm{e}}^{\mathrm{o}}\right|}<10^{-4}
$$

Hence, the number of central and edge users in every cell will be chosen in a dynamic way.

\subsection{Pilot Scheduling}

Here, the asymptotic orthogonality among 2 arbitrary vectors is modeled. Let the 2 self-governing arbitrary vectors $\mathrm{j}, \mathrm{i} \in \mathrm{G}^{\mathrm{N} \times 1}$ indicates the 2 self-governing arbitrary vectors with the distribution function $\mathrm{Q}\left(0, \mathrm{vH}_{\mathrm{B}}\right)$ and is stated as,

$$
\lim _{B \rightarrow \infty} \frac{\mathrm{j}^{+} \mathrm{i}}{\mathrm{B}} \rightarrow 0 \text { and } \lim _{\mathrm{B} \rightarrow \infty} \frac{\mathrm{j}^{+} \mathrm{j}}{\mathrm{B}} \stackrel{\mathrm{\kappa}}{\rightarrow} \mathrm{v}
$$

whereas, the variable $\kappa$ indicates the approximately time convergence.

\subsection{Optimal Pilot Scheduling Exploiting BPSO}

The major contribution of this paper is to propose a technique in a massive-MIMO system to decrease pilot contagion. By exploiting BPSO, the pilot scheduling method is carried out for pilot scheduling.

The PSO is on the basis of the swarm intelligence with real values [10]. In order to enable PSO to search for optimal values in a given search space, there is necessitate of a modulation that assists in the modulation of real values into binary form. Moreover, similar is stated in eq. (22).

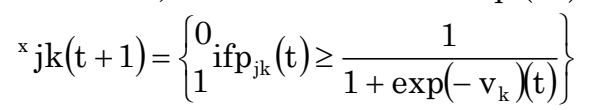

In eq. (22), $\mathrm{p}_{\mathrm{jk}}(\mathrm{t})$ denotes determining the probability of node $\mathrm{j}$

\section{Result and Analysis}

The performance evaluation of the adopted technique was presented in this section, and it was verified by variation the users, transmission power, antennas in Base Station, and log-normal shadowing fading. On basis of performance metrics, the analysis was performed.

The performance analysis of the proposed and conventional models regarding the SINR and achievable rate parameters are summarized in Tables 1,2,3, and 4 . The overall analysis states that the maximum SINR and achievable rate is obtained by the proposed method than the conventional methods.

Table 1: Performance Analysis on The Basis of Number of Users

\begin{tabular}{lll}
\hline Techniques & SINR(dB) & Achievable rate $(\mathrm{bps} / \mathrm{Hz})$ \\
\hline PSO & 98.44 & 42.46 \\
GA & 309.64 & 46.43 \\
ABC & 334.48 & 48.99 \\
ESMO & 338.38 & 49.25 \\
Proposed Method & $\mathbf{3 2 4 . 4 3}$ & $\mathbf{4 9 . 7 9}$ \\
\hline
\end{tabular}


Table 2: Performance Analysis on The Basis of Number of Antenna In BS

\begin{tabular}{lll}
\hline Techniques & SINR(dB) & Achievable rate $(\mathbf{b p s} / \mathrm{Hz})$ \\
\hline PSO & 27.37 & 6.277 \\
GA & 26.77 & 6.782 \\
ABC & 27.73 & 7.077 \\
ESMO & 27.87 & 7.229 \\
Proposed Method & $\mathbf{2 8 . 6 7}$ & $\mathbf{7 . 6 6 8}$ \\
\hline
\end{tabular}

Table 3: Performance Analysis on the basis of Transmission Power

\begin{tabular}{lll}
\hline Techniques & Achievable rate $(\mathrm{bps} / \mathrm{Hz})$ & SINR(dB) \\
\hline PSO & 6.601 & 16.12 \\
GA & 6.689 & 12.91 \\
ABC & 6.879 & 16.65 \\
ESMO & 6.988 & 16.79 \\
Proposed Method & 7.602 & 17.56 \\
\hline
\end{tabular}

Table 4: Performance Analysis ON THE BASIS OF Shadow Fading

\begin{tabular}{lll}
\hline Techniques & SINR(dB) & Achievable rate $(\mathbf{b p s} / \mathbf{H z})$ \\
\hline PSO & 26.02 & 6.424 \\
GA & 22.88 & 6.855 \\
ABC & 26.56 & 6.958 \\
ESMO & 26.62 & 4.22 \\
Proposed Method & $\mathbf{2 7 . 5 6}$ & 7.476 \\
\hline
\end{tabular}

\section{Conclusion}

This work proposes the grouping of user techniques to reduce the pilot contagion. The developed grouping of user method was developed to group users into the edge and a center user on the basis of the three parameters and it was important to comprehend that grouping of user possess effects on pilot scheduling. Therefore, it was important to model an effectual grouping of user techniques to enhance the capacity of the system. Hence, the sparse FCM was developed for grouping of the user to allocating the similar pilot series to center users. The developed clustering technique used SINR, large-scale fading factor, and the distance of user as decision parameters rather than the standard distance measure in sparse FCM, so that number of edge, and center users in each cell, was chosen in a dynamic way. Hence, the developed grouping of users shuns from the unsuitable users grouping, set up the efficient grouping in most awful circumstances of the channel. Next, pilot scheduling was carried out on the basis of the BPSO. The BPSO was used to assign an optimal pilot series to the users which degrade from maximum degradation.

\section{Compliance with Ethical Standards}

Conflicts of interest: Authors declared that they have no conflict of interest.

Human participants: The conducted research follows the ethical standards and the authors ensured that they have not conducted any studies with human participants or animals.

\section{References}

[1] Parisa PasangiMahmoud AtashbarMahmood Mohassel Feghhi,"Blind downlink channel estimation of multi-user multi-cell massive MIMO system in presence of the pilot contamination", AEU - International Journal of Electronics and Communications6 February 2020.

[2] Awais Ahmed, Muhammad ZiaIhsan ul Haq,"Secret key acquisition under pilot contamination attack", AEU International Journal of Electronics and Communications17 August 2019.

[3] Muhammad HassanMuhammad ZiaNaeem Bhatti,"Pilot contamination attack detection for multi-cell MUmassive MIMO system", AEU - International Journal of Electronics and Communications21 October 2019.

[4] Jiancun FanWeiqi LiYing Zhang,"Pilot contamination mitigation by fractional pilot reuse with threshold optimization in massive MIMO systems", Digital Signal Processing22 March 2018.

[5] Satyasen Panda,"Performance improvement of Massive MIMO system under rapid user mobility conditions", Computer Communications26 March 2020.

[6] Omar A. Saraereh, Imran Khan,Byung Moo Lee, and Ashraf Tahat, "Efficient Pilot Decontamination Schemes in 5G Massive MIMO Systems", Electronics, vol.8, no.1, pp.55, 2019. 
Pilot scheduling to mitigate pilot contamination using optimization algorithm in Massive MIMO systems

[7] Xiangyu Chang ; Qingnan Wang ; Yuewen Liu ; Yu Wang, "Sparse Regularization in Fuzzy c-Means for HighDimensional Data Clustering", IEEE Transactions on Cybernetics, vol.47, no.9, pp.2616 - 2627, September 2017.

[8] Amin Khansefid, and Hlaing Minn,"On Channel Estimation for Massive MIMO with Pilot Contamination", Communications Letters, volume.19, number.9, page no.1660-1663, 2015.

[9] Daniel C. Araujo, TarasMaksymyuk, Andre L. F. de Almeida, Tarcisio Maciel, Joao C. M. Mota, and Minho Jo, "Massive MIMO: Survey and Future Research Topics", IET Communications, volume.10, number.15, page no.1938-1946, 2016.

[10] V. Alappatt and P. M. Joe Prathap, "A Hybrid Approach Using Ant Colony Optimization and Binary Particle Swarm Optimization (ACO: BPSO) for Energy Efficient Multi-path Routing in MANET," 2020 Advanced Computing and Communication Technologies for High Performance Applications (ACCTHPA), Cochin, India, pp. $175-178,2020$

[11] Amol V Dhumane,"Examining User Experience of eLearning Systems using EKhool Learners",Journal of Networking and Communication Systems (JNACS), Volume 3, Issue 4, October 2020. 